\section{IN BRIEF}

\section{$\Rightarrow$ SYSTEMIC LUPUS ERYTHEMATOSUS}

\section{Gut microbiota linked to kidney disease in SLE}

Distinct patterns of gut microbiome dysbiosis correlated with disease activity in a study of 61 female patients with systemic lupus erythematosus (SLE). The results of faecal 16S rRNA analysis were confirmed in two independent SLE cohorts and revealed a fivefold increase in the amount of Ruminococcus gnavus in patients with SLE compared with healthy individuals. Expanded populations of $R$. gnavus were particularly prevalent in patients with lupus nephritis; individuals with active nephritis also had high serum concentrations of antibodies against cell wall lipoglycans from certain strains of $R$. gnavus.

ORIGINAL ARTICLE Azzouz, D. et al. Lupus nephritis is linked to disease-activity associated expansions and immunity to a gut commensal. Ann. Rheum. Dis. https://doi.org/10.1136/annrheumdis-2018-214856 (2019)

\section{VASCULITIS}

\section{Tocilizumab improves quality of life in GCA}

Analysis of health-related quality of life (HR-QOL) data from the GiACTA trial, in which 251 patients with giant cell arteritis (GCA) were treated with either tocilizumab or placebo in addition to a tapered dose of prednisone, showed improvements in overall HR-QOL for patients treated with tocilizumab plus prednisone compared with those treated with placebo plus prednisone. Clinically meaningful differences were reported for 36-Item Short-Form Health Survey Mental Component Summary scores $(P=0.0012)$ and for Patient Global Assessment scores $(P=0.0029)$ in those receiving tocilizumab. ORIGINAL ARTICLE Strand, $V$. et al. Health-related quality of life in patients with giant cell arteritis treated with tocilizumab in a phase 3 randomised controlled trial. Arthritis Res. Ther. 21, 64 (2019)

\section{RHEUMATOID ARTHRITIS}

\section{Gut microbiota changes pre-date onset of RA}

A comparison of the composition of the gut microbiota of 50 first-degree relatives of patients with rheumatoid arthritis (RA; autoantibody-negative and asymptomatic) and 83 individuals with pre-clinical RA (autoantibody-positive and/or symptomatic) revealed a significant enrichment of Prevotella species in the guts of those with pre-clinical RA $(P=0.04)$. These results indicate that increases in the prevalence of Prevotella species, previously noted in the guts of newly diagnosed patients with RA, could actually precede the onset of disease, and lend support to the mucosal origins hypothesis for RA.

ORIGINAL ARTICLE Alpizar-Rodriguez, D. et al. Prevotella copri in individuals at risk for rheumatoid arthritis. Ann. Rheum. Dis. https://doi.org/10.1136/annrheumdis-2018-214514 (2019)

\section{$\Rightarrow$ THERAPY}

\section{No need for DMARD holiday before arthroplasty}

Administration of intravenous abatacept $<4$ weeks before knee or hip arthroplasty was not associated with an increased risk of post-operative infection compared with halting therapy $>4$ weeks before surgery in a study of 1,780 patients with rheumatoid arthritis (RA). No significant differences were seen in the rates of hospitalized infection (total and non-urinary), 1-year prosthetic joint infection or 30-day readmission between patients who received abatacept $<4$ weeks or $>4$ weeks before surgery; however, glucocorticoid use during the 3 months prior to surgery was associated with an increased risk of infection.

ORIGINAL ARTICLE George, M. D. et al. Timing of abatacept before elective arthroplasty and risk of post-operative outcomes. Arthritis Care Res. https://doi.org/10.1002/acr.23843 (2019)

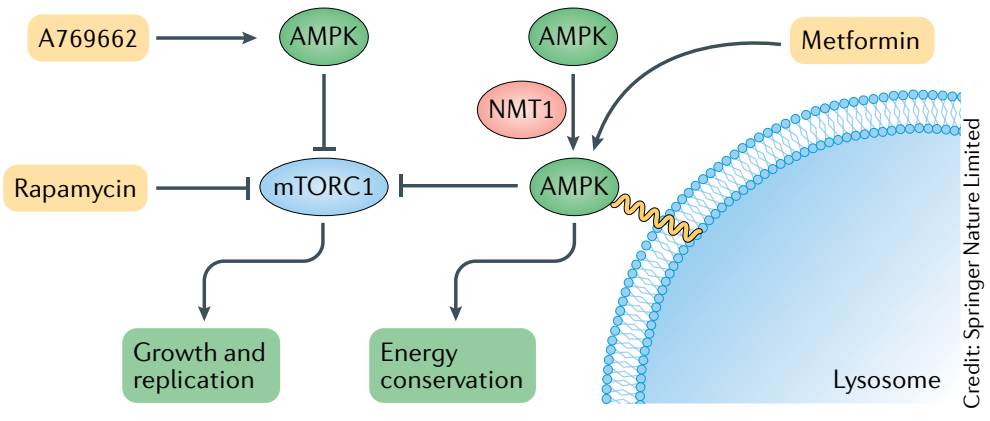

IMMUNOMETABOLISM

\title{
AMPK: a therapeutic target in RA?
}

In rheumatoid arthritis (RA), T cells have an altered metabolic profile in which glucose is shifted from glycolysis and ATP production (an energy-conserving programme) to the pentose phosphate pathway and biosynthesis (a cell-building programme). This rerouting of glucose leads to a tissue-invasive and pro-inflammatory phenotype in T cells that is thought to drive chronic inflammation. In a new study, researchers implicate dysfunctional lysosomal recruitment of energy sensor AMP-activated protein kinase (AMPK) in this process.

"To coordinate energy supply and proliferative behaviour, cells use AMPK to sense their energy resources," explains corresponding author Cornelia Weyand. "AMPK is activated when AMP/ATP and $\mathrm{ADP} / \mathrm{ATP}$ ratios rise and it restores energy balance by switching on catabolic processes to produce ATP and switching off anabolic processes that consume ATP."

"The counter player of AMPK, the mammalian target of rapamycin (mTOR), integrates growth factor signals with nutrient sensing to promote cellular replication and growth," says Weyand. "Importantly, these two systems are interconnected: under low-energy conditions, AMPK inactivates mTORC1." The function of AMPK is dependent on its translocation to the lysosome, which requires $\mathrm{N}$-myristoylation of its $\beta 1$ and $\beta 2$ subunits, catalyzed by $\mathrm{N}$-myristoyltransferase (NMT).

In the new study, the researchers detected defective $\mathrm{N}$-myristoylation in $\mathrm{CD}^{+} \mathrm{T}$ cells from patients with RA (hereafter referred to as
RA T cells). RA T cells had lower concentrations of NMT1 than T cells from healthy individuals or patients with other autoimmune diseases. This reduced NMT1 abundance was associated with increased T helper $1\left(\mathrm{~T}_{\mathrm{H}} 1\right)$ cell and $\mathrm{T}_{\mathrm{H}} 17$ cell differentiation in vitro, which could be mimicked by silencing NMT1 in healthy $\mathrm{T}$ cells or suppressed by forcing NMT1 overexpression.

"Loss-of-function of NMT1 biased $\mathrm{T}$ cells to cause synovial inflammation in a model system of human synovial tissue engrafted into immune-deficient mice," reports Weyand. "By contrast, gain-offunction of NMT1 in RA T cells was sufficient to suppress synovial inflammation in this model."

The researchers found that NMT1 deficiency prevented lysosomal recruitment and activation of AMPK, resulting in unrestrained mTORC1 signalling. Administration of an AMPK activator that functions independently of the lysosomal pathway (A769662), but not of metformin (an AMPK activator that functions through the lysosomal pathway), inhibited tissue inflammation in the human-synovium chimera model, and had comparable effects to mTORC1 inhibitor rapamycin.

"We have essentially identified a new therapeutic target and we are planning clinical trials that bring this strategy from the bench to the bedside," concludes Weyand.

Jessica McHugh

ORIGINAL ARTICLE Wen, Z. et al.

$\mathrm{N}$-myristoyltransferase deficiency impairs activation of kinase AMPK and promotes synovial tissue inflammation. Nat. Immunol. 20, 313-325 (2019) 